\title{
Transparent glass slab thickness measurement by binary- spot focus signal
}

\section{Medición de espesor de placas de vidrio por señal de error de foco de spot binario}

\author{
A. D. Aguilar ${ }^{1 *}$, J. R. Torga ${ }^{1,2}$ \\ 1. Universidad Tecnológica Nacional - Facultad Regional Delta, Ciudad de Campana, Provincia de Buenos \\ Aires, Argentina \\ 2. Consejo Nacional de Investigaciones Científicas y Técnicas, sede GIOL, Ciudad Autónoma de Buenos Aires, \\ Argentina \\ (๗) E-mail: adaguilar@frd.utn.edu.ar.com / ad.agbur@yahoo.com
}

Received: 14/11/2019 Accepted: 12/12/2019

DOI: 10.7149/OPA.52.4.51035

\begin{abstract}
:
Focus error systems based on the astigmatic principle relying on four quadrant photo detectors as the signal generation element have widely been used in many different applications. Nevertheless, its working principle is not particularly suitable for measurements involving more than one spot. In this work we propose a new method that uses a CCD as the detection device as well as a method of processing acquired data. A side effect of this change is the loss of the FES parameter key to these techniques. To overcome this issue a new parameter named focus signal is proposed. The thickness measuring process of a transparent sample with two reflective layers using this technique is also presented.
\end{abstract}

Key words: Focus-Error-Signal, astigmatic, thickness, beam profilometer, multiple spots

\section{RESUMEN:}

Los sistemas de error de foco basados en el principio astigmático que utilizan detectores de cuatro cuadrantes como elemento generador de señal han sido usados ampliamente en una gran variedad de aplicaciones. Sin embargo, su principio de funcionamiento no es particularmente adecuado para medidas que involucran más de un spot. En este trabajo proponemos un nuevo método que usa un CCD como dispositivo de detección y un método de procesar los datos adquiridos. Un efecto secundario de este cambio es la pérdida del parámetro $S E F$, clave en estas técnicas. Para superar este problema se propone un nuevo parámetro que llamamos señal de foco. El proceso de medición de espesor de una muestra transparente con dos caras reflectoras usando esta técnica también es presentado.

Palabras clave: Señal de error de foco, astigmatismo, espesor, perfilómetro de haz, múltiples spots

\section{REFERENCES AND LINKS / REFERENCIAS Y ENLACES}

[1] C. Bricot, J.C. Lehureau, C. Puech, F.L. Carvennec, “Optical Readout of Videodisc”, IEEE Transactions on Consumer Electronics, 8 (8), 304-308 (1976),

[2] B. Hnilička, A. Voda, H.-J. Schröder, "Modelling the characteristics of a photodetector in a DVD player", Sensors and Actuators A: Physical, 120 (2), 494-506 (2005).

[3] S. Goldwasser, "Lasers in Consumer Electronics: The Optical Pickup", Optics and Photonics News, 22 (2), 12 (2011)

[4] C.-H. Liu, Z.-H. Li, "Application of the astigmatic method to the thickness measurement of glass substrates”, Appl. Opt., AO, 47 (21), 3968-3972 (2008). 
[5] C.-H. Liu, S.-C. Yeh, H.-L. Huang, "Thickness measurement system for transparent plates using dual digital versatile disc (DVD) pickups", Appl. Opt., A0, 49 (4), 637-643 (2010).

[6] Z. Bai, J. Wei, "Focusing error detection based on astigmatic method with a double cylindrical lens group", Optics \& Laser Technology, 106, 145-151 (2018).

[7] K.C. Fan, C.Y. Lin, L.H. Shyu, "The development of a low-cost focusing probe for profile measurement", Meas. Sci. Technol. 11 (1), N1-N7 (1999).

[8] E.A. Domené, D. Schiltz, D. Patel, T. Day, E. Jankowska, O.E. Martínez, J.J. Rocca, C.S. Menoni, “Thin film absorption characterization by focus error thermal lensing”, Review of Scientific Instruments. 88 (12), 123104 (2017).

[9] C.-S. Liu, T.-Y. Wang, Y.-T. Chen, "Novel system for simultaneously measuring the thickness and refractive index of a transparent plate with two optical paths", Appl. Phys. B., 124 (9), 180 (2018).

[10] T. Coleman, Y. Li, "An Interior Trust Region Approach for Nonlinear Minimization Subject to Bounds”, SIAM J. Optim., 6 (2), 418-445 (1996).

[11] T.F. Coleman, Y. Li, "On the convergence of interior-reflective Newton methods for nonlinear minimization subject to bounds", Mathematical Programming., 67 (1), 189-224 (1994).

[12] L. Li, C. Kuang, D. Luo, X. Liu, "Axial nanodisplacement measurement based on astigmatism effect of crossed cylindrical lenses”, Appl. Opt., A0., 51 (13), 2379-2387 (2012).

[13] F. Arnesson, How to run a semiconductor diode laser in a stable way, Master Thesis, Umeå, Umeå Univeristet, (2012).

[14] S. Nemoto, "Waist shift of a Gaussian beam by plane dielectric interfaces", Appl. Opt., AO., 27 (9), 1833-1839 (1988).

[15] M. N. Polyanskiy, "Refractive index database, Refractive index of BK7 - SCHOTT, (n.d.)". (accessed June 25, 2019).

[16] E.N. Morel, M.V. Gutierrez, H.M. Miranda, E.L. Sambrano, J.R. Torga, “Optical coherence tomographybased scanning system for shape determination, wall thickness mapping, and inner inspection of glass containers", Appl. Opt., A0., 52 (9), 1793-1798 (2013).

\section{Introduction}

Focus error signal, autofocus or auto tracking share a configuration based on the astigmatic principle first published in 1976 [1], [2] and have since been used in a great variety of consumer electronic products [3]. These kinds of systems have turned to be very effective in autofocus applications in diverse areas such as maskless laser lithography, imaging, recording and retrieval of data in optical storage media. The application of this technique to thickness measurement of transparent plates has been recently reported by Liu [4] where an astigmatic optical system was used to measure the thickness of a glass slab based in the shift in the position of the waist of a Gaussian beam due to a change in the refractive index in the beam path by inserting the slab between an astigmatic lens group and a four quadrant detector. The same author proposes an alternative method using two DVD pick-up heads [5], one as transmitter and the other as receptor.

In most of the papers mentioned the configuration is simple, the astigmatic detection is achieved using a single cylindrical lens and a quadrant photodetector. In all cases, the basic principle is the same: depending on the beam path, the cylindrical lenses modify the intensity distribution which results in a change in the FES (focus error signal) value [6]. Therefore, by having a calibrated S-curve it is possible to measure a change in sample position with respect to the objective lens focal position.

Commonly, the FES technique is adequate for samples with only one reflective surface, either measuring the position of the surface [7], surface variations with time [8] or changes in the optical path of the beam [4].

In this paper we present a variant using two cylindrical lenses in a setup similar to that proposed in [6] but using a CCD as the detection element instead. This modification allows us to measure, simultaneously, more than one reflection of the sample, thus extending the possible applications of the astigmatic principle. We demonstrate the viability of the technique by measuring the position of the anterior and posterior reflections from air-glass and glass-air interfaces of a glass slab and ultimately obtaining the thickness of the sample. The resulting setup performed satisfactorily and provides a very simple, low cost solution to the thickness measurement of transparent plate and can be custom designed to accommodate a wide range of thickness from $\ll 1 \mathrm{~mm}$ to several millimetres as opposed to other techniques that can be 
applied to either smaller values, the case of ellipsometry, or millimetre-sized values, triangulation methods [9]. We found this system particularly suitable for use in off-line quality control.

\section{Setup}

The setup used in this work is shown in Figure 1, the light source is a laser (Thorlabs LP642-SF20) coupled into a single-mode fibre; the output beam is collimated with a microscope objective (CO, Olympus PlanN; 20x/0.40; $\infty / 0.17 / F N 22)$. A beam splitter followed by a bi-convex spherical lens (OBJ, LB1757-A, $30 \mathrm{~mm}$ focal length) which focuses the beam onto the sample. The reflected beam is directed towards the detector after passing through two plano-convex cylindrical lenses of $50 \mathrm{~mm}$ focal length (Edmund Optics \#69-779) forming a mutually orthogonal double cylindrical lens group (LCA and LCB). Contrary to the commonly used four quadrant photodetector, we used a CCD camera or a beam profilometer (Newport LBP2-HR-VIS2) instead. The distances between elements were measured using a vernier calliper and then the total distance was added, they are summarised in Table 1.

TABLE 1. Distances between setup elements [mm]

\begin{tabular}{|c|c|}
\hline CO-OBJ & 102.5 \\
\hline OBJ-LCA & 180.46 \\
\hline LCA-LCB & 79.5 \\
\hline LCB-DET & 182.74 \\
\hline
\end{tabular}

Distances between collimator microscope objective (CO) and the objective lens (OBJ) were mostly constrained by optomechanical gear, while the positions of LCA, LCB and DET were determined iteratively until a suitable spot size was obtained across the measurement range.

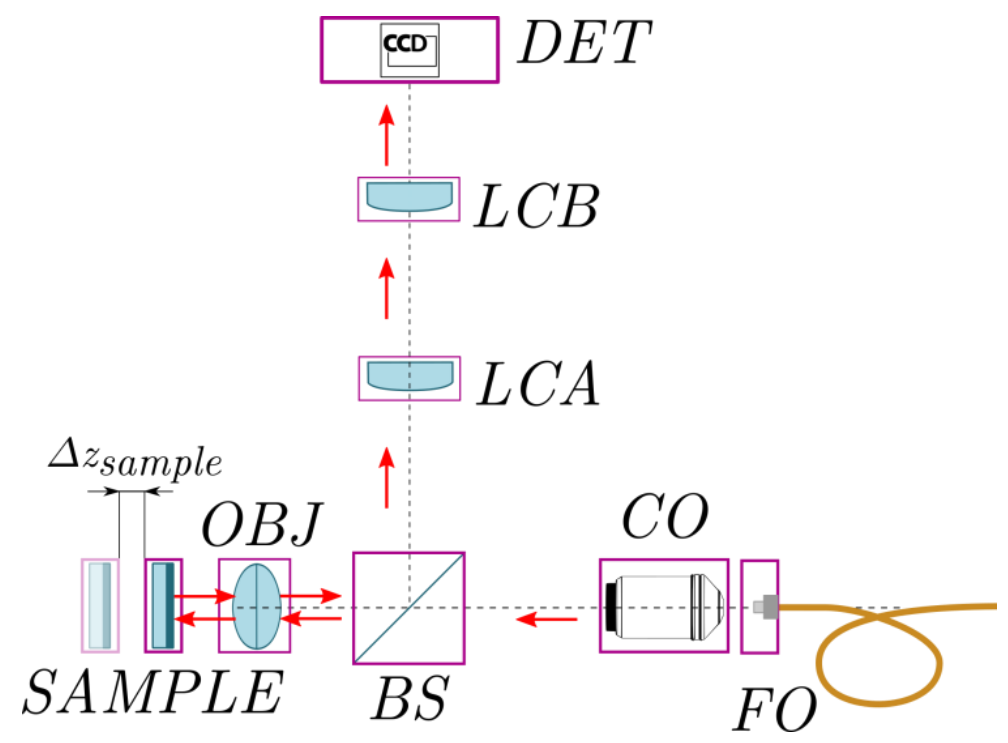

Fig.- 1 Experimental setup layout. FO- Optical fibre output, CO- Microscope objective collimator, BS- beam splitter, OBJ- Objective lens, LCA- First Cylindrical lens, LCBSecond cylindrical lens, DET- Beam profilometer. 


\section{Binary-spot focus signal}

The central purpose of this work is to show that the replacement of the four quadrant photodiode for a CCD gives the possibility of detecting two surfaces simultaneously and extending the sensitivity of the technique given the increase in the number of pixels used to obtain each measurement. On the other hand the response time of the detector is impaired, but for the type of applications proposed in this work this does not constitute any limitation. A typical intensity profile generated with the experimental setup described in the previous section, in the case of a sample with a single surface, is shown on Figure 2(b). The $x^{\prime}$ and $y^{\prime}$ axes are chosen coinciding with the main axes of the ellipse, while $x$ and $y$ are the fixed axes to the detector system. In this scheme $\omega_{x}$, and $\omega_{y}$, represent the usual definitions of the beam waist in the $x^{\prime}$ and $y^{\prime}$ axes respectively. Due to the nature of the four quadrant photodiode normally used in conventional FES system, when two or more reflections are focused on the detector simultaneously, an erroneous FESis obtained. This happens if the intensity profile obtained in the detector, due to reflections from both surfaces, named $A$ and $B$ throughout this paper, are within the range of the system simultaneously as depicted on Figure 2(a). In this example $\omega_{x}^{A} \approx \omega_{y}^{B}$ and $\omega_{y}^{A} \approx \omega_{x}^{B}$, thus, resulting in a FES value close to zero when neither spot has a shape that would produce such a result. Moreover, in a situation in which two or more spots of similar sizes $\left(\omega_{x}^{A} \approx \omega_{x}^{i}\right.$ and $\left.\omega_{y}^{A} \approx \omega_{y}^{i}\right)$ overlay, it would not be possible to distinguish the $F E S$ value for each spot individually.

Our goal then was to identify the characteristics of a binary-spot produced by the superposition of two beams reaching the detector simultaneously. To achieve this we proposed a new detection scheme and an alternative processing algorithm to the traditional one used in the focus error signal systems. Firstly, we replaced the quadrant photodetector for a CCD and then each acquired frame was processed to obtain the spot parameters individually. This processing consisted in fitting a bivariate Gaussian, Equation 1, to each spot in the frame using nonlinear least squares [10,11].
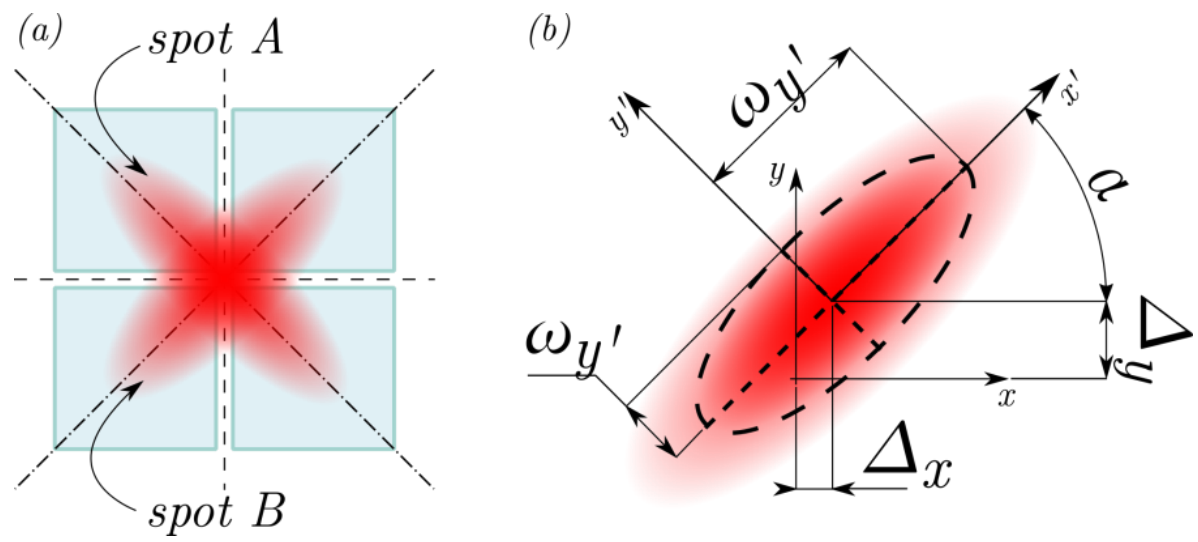

Fig.- 2(a) drawing depicting a binary spot configuration (over a four quadrant detector) that renders the traditional FES unusable. (b) Drawing of a Gaussian spot with constants used in Equation 1. The apostrophe makes the distinction between the spot's own coordinate axis, $\mathrm{x}^{\prime}$ and $\mathrm{y}^{\prime}$, and the detectors coordinates, $\mathrm{x}$ and $\mathrm{y}$.

$$
I_{(x, y)}=I_{0} e^{-2\left[\frac{\left((x \cos (\alpha)-y \sin (\alpha))-\Delta_{x}\right)^{2}}{\omega_{x}{ }^{2}}+\frac{\left((x \sin (\alpha)-y \cos (\alpha))-\Delta_{y}\right)^{2}}{\omega_{y \prime^{\prime}}}\right]}
$$

Constants in Equation 1:

- $I_{0}$ : amplitude of intensity distribution

- $\omega_{x \prime / y^{\prime}}:$ waist size in $x^{\prime}$ and $y^{\prime}$ direction.

- $\Delta_{x / y}$ : shift of the centre of the distribution.

- $\alpha$ : inclination of the $x^{\prime} / y^{\prime}$ axes.

- $\quad x / y$ and $x^{\prime} / y^{\prime}$ are the detector-fixed coordinates and beam-fixed coordinates respectively. 
Replacing the quadrant photodiode means the naturally generated $F E S$ is no longer available. As an alternative we proposed a new parameter called focus signal $(S F)$, defined as shown in Equation 2, as the main descriptor of the system.

$$
S F=\frac{\omega_{x \prime}-\omega_{y \prime}}{\omega_{x \prime}+\omega_{y \prime}}
$$

In which $\omega_{x \prime}$ and $\omega_{y}$, are the waists of each spot measured on the orthogonal axes $x^{\prime}$ and $y^{\prime}$ (Figure 2(b)). $S F$ has two extrema one positive and one negative, that tend to +1 and -1 as the spot gets slender. The closer the $S F$ approaches these extrema, the higher the sensitivity of the system. Traditional FES systems may reach these values since they use real quadrant detectors and the gap between quadrants may be large enough to effectively produce a value of 0 for a pair of opposite quadrants. The system proposed in this paper however can only produce a $S F$ that approximates these extrema as the waists will be diffraction limited at best.

In the following sections we describe the process employed to measure the thickness of a transparent sample with the optical system described in Section 2 and $S F$ as defined in Equation 2.

\section{Experiment}

To demonstrate our hypothesis we carried out an experiment consisting of two stages, first a calibration of the $S F$ and second a measurement of a sample.

\section{4.a. Calibration}

In order to establish a relationship between the SF and the position of a sample reflection within the measurement range we performed a calibration sequence by moving a surface of the same material as the sample but thick enough so that the second reflection is not within the measurement range, while recording the frames along with the position, $\left(Z_{\text {sample }}\right.$ in Figure 1$)$, as reported by the motion control system (positioning was achieved using an ESP-301 controller and a LTAHSPPV6 motor, Newport). The sequence was composed of 46 frames acquired by using the profilometer and automatically maximising the dynamic range for each frame.

(a)

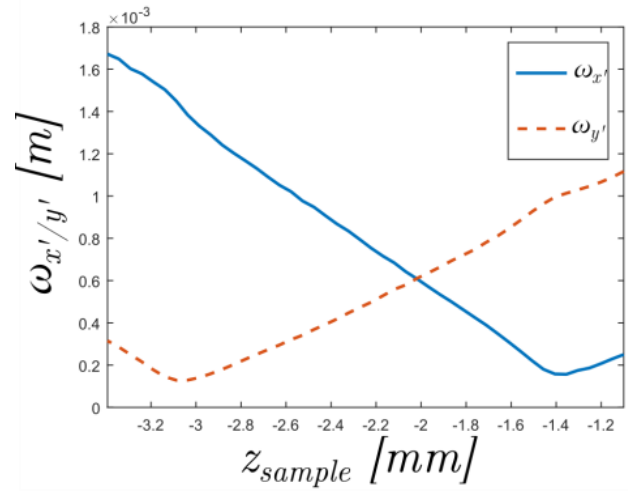

(b)

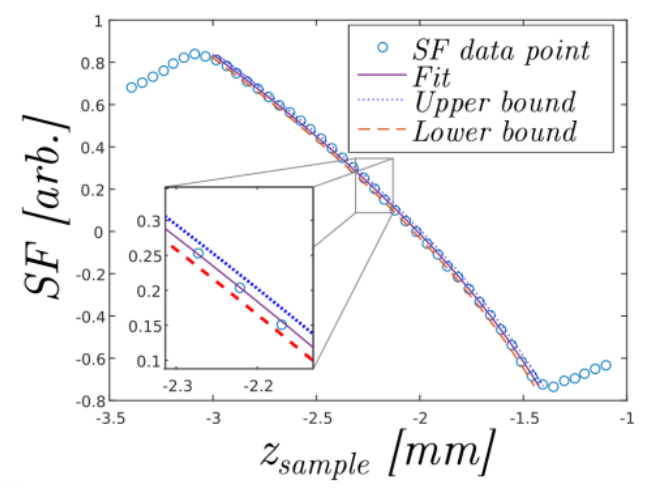

Fig.- 3(a): beam profile, continuous line is $\omega_{\mathrm{x}}$, and dashed line is $\omega_{y^{\prime}}$ (b): calculated, inset: detail showing data points, fit and fit bounds. 
After fitting each frame with Equation 1, the values of $\omega_{x}$, and $\omega_{y}$, were obtained and used to calculate the focus signal $S F$ (Equation 2) along a range of $2.3 \mathrm{~mm}$. The $S F$ curve presented a measurement range of $1.7 \mathrm{~mm}$, suitable for the thickness of the samples considered in this paper. In this region the $S F$ exhibited a maximum value of 0.837 and a minimum of -0.732 . Additionally the measurement interval was fitted with a second grade polynomial to calculate the $Z$ value of each reflection, the coefficients of the polynomial in Equation 3 are detailed in Table 2. The beam profiles and S-curve are shown in Figure 3.

$$
Z_{(S F)}=A S F^{2}+B S F+C
$$

TABLE 2 Equation and parameters for SF fit.

\begin{tabular}{|c|c|}
\hline Coefficient & Value $\begin{array}{c}\text { +upperbound } \\
\text {-lowerbound }\end{array}$ \\
\hline $\mathrm{A}$ & $-0.2435_{-0.0177}^{+0.0178}$ \\
\hline $\mathrm{B}$ & $-0.9792_{-0.0077}^{+0.0078}$ \\
\hline $\mathrm{C}$ & $-2.011_{-0.005}^{+0.006}$ \\
\hline
\end{tabular}

The maximum error, in absolute value, between the recorded $Z_{\text {sample }}$ and the calculated $Z_{(S F)}$ was 13.2 $\mu \mathrm{m}$ and the standard deviation $(\sigma) 7.2 \mu \mathrm{m}$.

\section{4.b. Comment on range and resolution}

Focus error systems are known for their simplicity and high resolution capabilities, since the use of high NA values in combination with appropriately selected astigmatic lenses can lead to a very narrow range of the FES and therefore extremely high resolutions. In the particular case we are presenting, the range needs be large enough to accommodate both reflections, that is roughly the thickness of the sample under study.

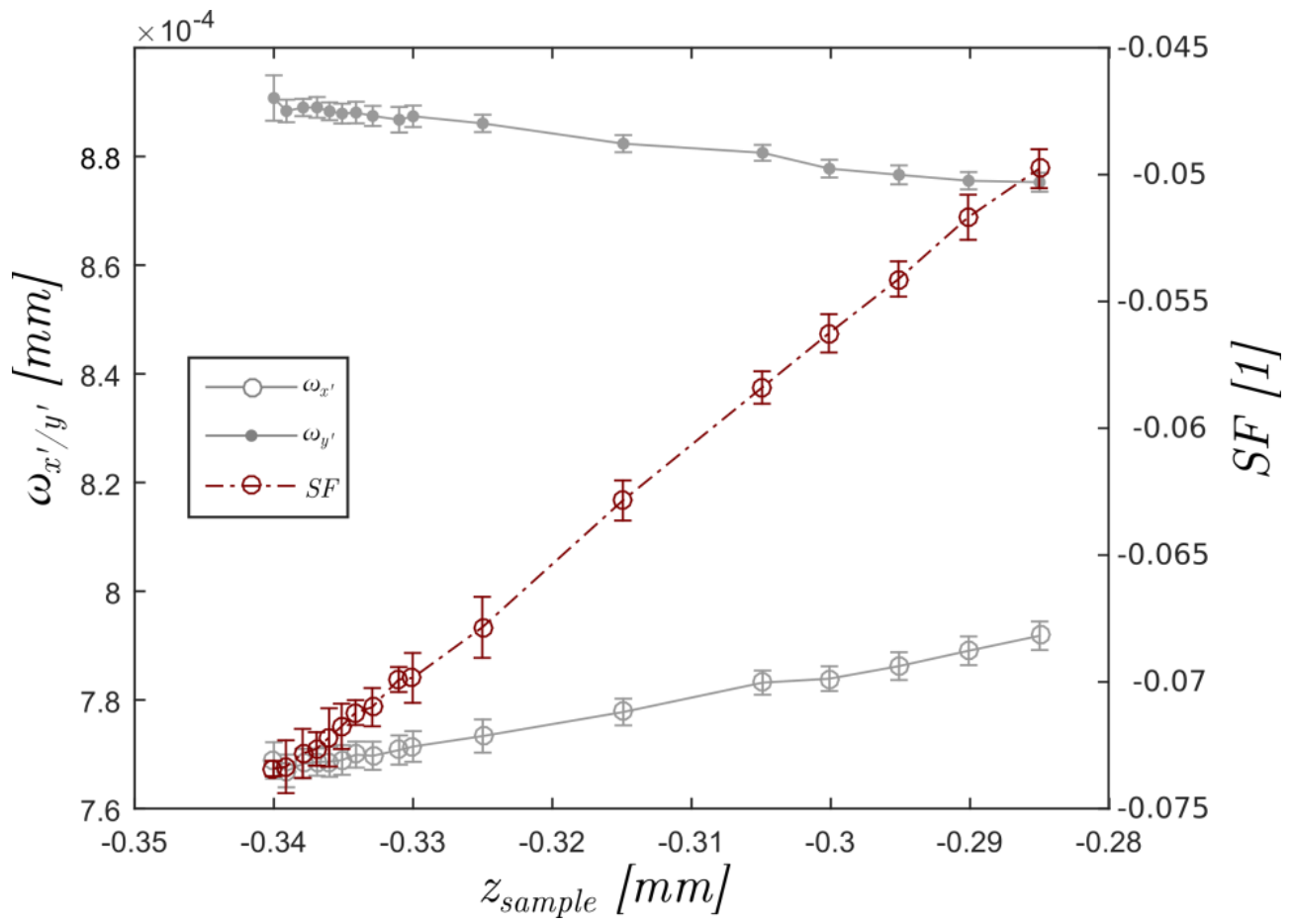

Fig.- 4 Two series of waists from close positions of the sample and the correspondent SF. Error bars represent the standard deviation of the set of nine frames acquired for each position, from which each waist value was obtained as the mean of the set. 
To assess the sensitivity of the method, the focus signal was measured at different positions of the sample along the $Z$ axis. In this case, only one side of the sample was measured. The measurements were made by making a series of steps ( 10 steps of $1 \mu \mathrm{m}$ and 7 steps of $5 \mu \mathrm{m}$ ) in a range of distances of $\approx 60 \mu \mathrm{m}$. For every position 9 frames were recorded, and the $S F$ calculated using the waist values extracted from them. It was found from the $S F$ that the first smaller steps overlap significantly and only the sixth point lies clearly outside the error bar of the first point, Figure 4, which means a step of approximately $5 \mu \mathrm{m}$ is the minimum step size for an unambiguous measurement of waist size. Nevertheless, as stated in [12] the resolution could be augmented by using higher lateral resolution CCDs, bits per pixel and better processing in order to be able to distinguish smaller changes spot size and therefore $S F$. For this calculation we considered the mean standard deviation of the $S F, \underline{\sigma} \simeq 0.0008$ and consequently, this uncertainty translated to distance using Equation 3 is $\underline{\sigma} \simeq 745 \mathrm{~nm}$ for a single data point. Considering this, an absolute error of $13.3 \mu \mathrm{m}$ for the $Z_{(S F)}$ from Equation 3 is quite conservative and can be further improved as well as the method to measure spot sizes, but this is out of the scope of this work.

\section{4.c. Thickness measurement}

The sample studied was a microscope slide which was positioned within the measurement region of the $S F$ and a set of 4 frames was recorded along the linear range obtained in the calibration. These frames contained the spots from reflections from frontal and posterior surfaces, A and B. That is to say, the total intensity recorded is actually the combination of two distinct intensity distributions.

$$
I_{(x, y)}=I_{(x, y)}^{A}+I_{(x, y)}^{B}
$$

During the experiments we operated the laser diode at an injection current lower than the nominal operating current in order to reduce its coherence and thus reduce the visibility of any eventual intensity modulation [13]. Therefore, and having observed no significant modulation, we omitted the interference term in Equation 4.

To extract the semi-axes of each of the spots a different fit was needed, by replacing Equation 1 in $A$ and $B$ terms in Equation 4, we obtain Equation 5:

$$
\begin{gathered}
I_{(x, y)}=I_{0}^{A} e^{-2 \frac{\left[\left(x \cos \left(\alpha^{A}\right)-y \operatorname{sen}\left(\alpha^{A}\right)\right)-\Delta_{x}^{A}\right]^{2}}{\left(\omega_{x}^{A}\right)^{2}}+\frac{\left[\left(x \operatorname{sen}\left(\alpha^{A}\right)-y \cos \left(\alpha^{A}\right)\right)-\Delta_{y}^{A}\right]^{2}}{\left(\omega_{y}^{A}\right)^{2}}} \\
+I_{0}^{B} e^{-2 \frac{\left[\left(x \cos \left(\alpha^{B}\right)-y \operatorname{sen}\left(\alpha^{B}\right)\right)-\Delta_{x}^{B}\right]^{2}}{\left(\omega_{x}^{B}\right)^{2}}+\frac{\left[\left(x \operatorname{sen}\left(\alpha^{B}\right)-y \cos \left(\alpha^{B}\right)\right)-\Delta_{y}^{B}\right]^{2}}{\left(\omega_{y}^{B}\right)^{2}}}
\end{gathered}
$$

The data in the binary-spot frames was fitted using this form, with the restriction that $\Delta_{x}{ }^{A}=\Delta_{x}^{B}$ and $\Delta_{y}^{A}=$ $\Delta_{y}^{B}$, and the same method as in the calibration frames. Consequently, two sets of semi-axes $\omega_{x \prime \prime}^{A}, \omega_{y^{\prime}}^{A}$ and $\omega_{x \prime}^{B}, \omega_{y \prime}^{B}$, were obtained from each frame and their respective $S F$ values were calculated.

\section{Results}

Using our calibrated focus signal fit we then obtained the corresponding position values $Z^{A}$ and $Z^{B}$ for $S F^{A}$ and $S F^{B}$ respectively, and so the difference between $Z^{A}$ and $Z^{B}$ is the calibration thickness.

$$
T_{C A L}=\left|Z^{A}-Z^{B}\right|
$$

This represents the distance between both reflective surfaces A and B assuming the medium between them is air, as it was during calibration, with an error of $\pm 0.013 \mathrm{~mm}$ from the fit of the $S F$ (Section 4.1). 


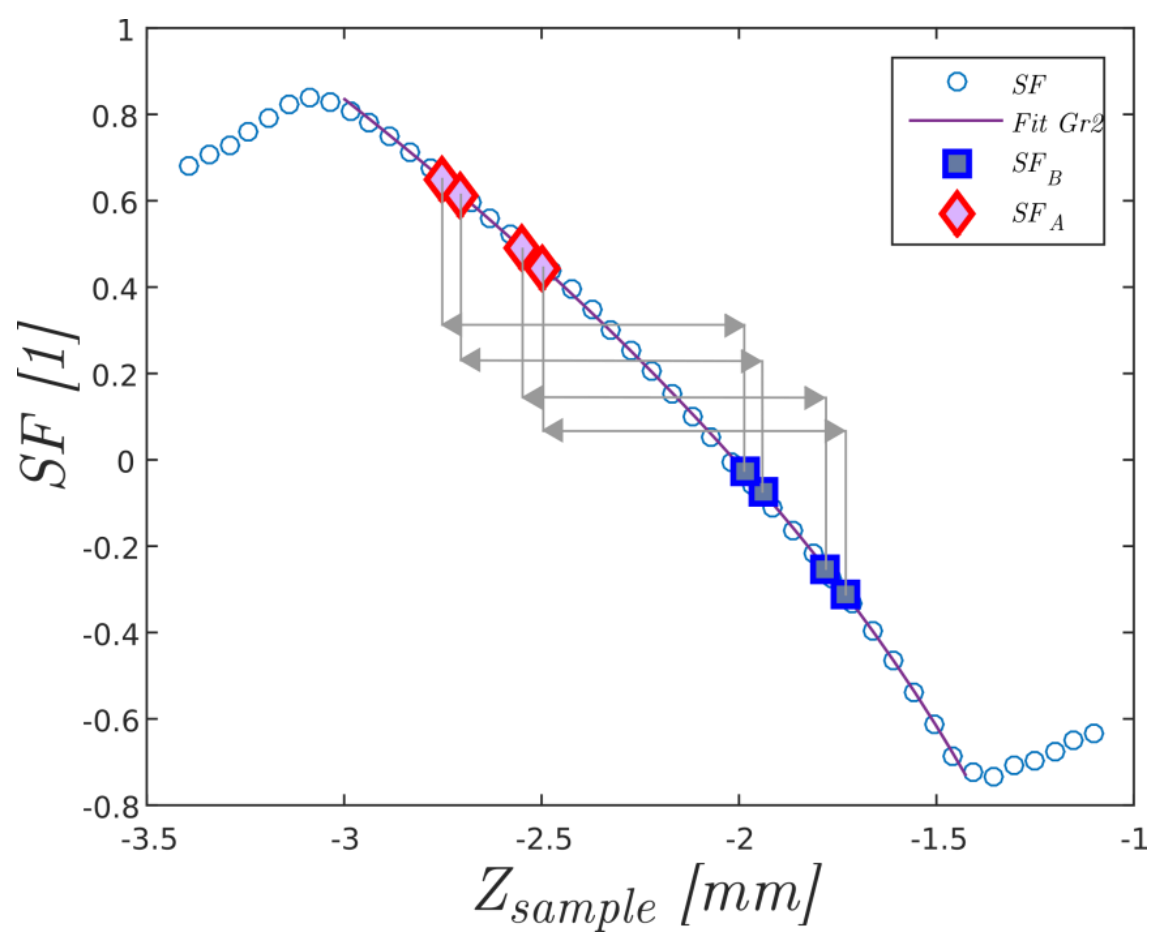

Fig.- 5 Detail of the results of the four recorded frames. SF values from surfaces A (rhombi) and B (squares) are plotted over the SF points (circles) and its fit.

To obtain the actual sample thickness we must consider the following: when we calibrated the system the beam travelled in air to the sample, and so the calibration $S F$ represents positions of a beam that gets reflected off a surface after travelling in air. However, when we measured the sample, one of the reflections corresponded to a beam that had travelled through air up to the first surface and continued through glass up to the posterior surface as shown in Figure 6.

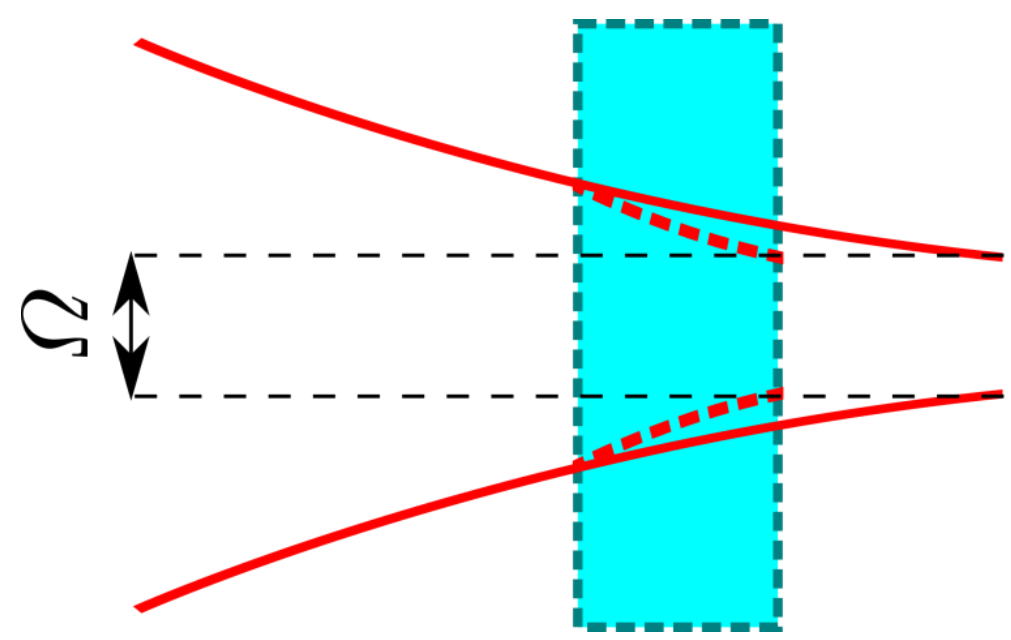

Fig.- 6 Diagram showing how a beam propagates differently in a denser optical medium and how it must travel further in air to reach the same spot diameter $\Omega$. Light is incident from the left. Incident beam propagating in air is depicted in continuous line and the beam propagating in glass-propagating in dashed line. The dashed bordered rectangle represents the measured slab. Note that only the first reflection was considered.

When this happens, the measuring beam travels a shorter distance $\left(n_{\text {glass }}>n_{\text {air }}\right)$ to reach a given beam diameter than the calibrating beam travelled in air to reach the same spot size. Thus, a correction is required to obtain the actual thickness. Following the work of S. Nemoto [14] we multiplied the calibration thickness by the index ratio: 


$$
\begin{gathered}
\begin{array}{c}
\text { ÓPTICA PURA Y APLICADA } \\
\text { www.sedoptica.es }
\end{array} \\
T_{G}=\frac{n_{\text {glass }}}{n_{\text {air }}} T_{C A L}
\end{gathered}
$$

With this correction we found four values of thickness considering $n_{\text {glass }}=1.5148 \pm 0.00005$ [15], thus, the propagated error in $T_{G}$ resulted in $0.0197 \mathrm{~mm}$. This was contrasted to an OCT measurement [16] which yielded a thickness of the slide of $1.167 \mathrm{~mm} \pm 5 \mu \mathrm{m}$. Additionally, another measurement was carried using a mechanical probe indicator which resulted in a thickness of $1.166 \mathrm{~mm}$ and $\sigma$ of $0.265 \mathrm{~mm}$ (over 21 repetitions).

TABLE 3 Thickness results summary, all dimensions in [mm]

\begin{tabular}{|c|c|c|c|c|c|c|c|}
\hline OCT & Probe Indicator & $T_{G 1}$ & $T_{G 2}$ & $T_{G 3}$ & $T_{G 4}$ & $\Delta T_{G}$ & $T_{G}$ \\
\hline $1.167 \pm 5 \mu \mathrm{m}$ & $1.166 \pm 265 \mu \mathrm{m}$ & 1.16 & 1.164 & 1.157 & 1.159 & \pm 0.0197 & 1.16 \\
\hline
\end{tabular}

\section{Conclusions}

We have demonstrated that thickness measurements can be performed using astigmatic detection systems that rely on reflected rather than transmitted light. Such a system was built and used to measure a microscope slide and results were compared to OCT measurements and found to be in agreement. The change of a quadrant detector for a CCD array enabled us to dissociate each reflection and recover the position of each surface and, ultimately, the sample thickness. The focus signal $(S F)$ parameter was introduced as an alternative to the traditional focus error signal (FES) and successfully applied to the thickness measurement. The use of a CCD comes with much higher latencies and processing demands compared to all analogue systems using quadrant detectors. This disadvantage, however, could be greatly reduced if a hardware, or even software optimised implementation of this method was used, such a system would be suitable for off-line analysis or slower phenomena, compared to traditional FES systems but still faster than the implementation presented in this paper.

\section{Acknowledgements}

Authors wish to acknowledge Pablo Tabla and Marcelo Sallese for their help in providing OCT measurements, Ana Laura Aguilar for her collaboration in proofreading and Ana Clara Aguilar for editing the text. This work was jointly financed by the Universidad Tecnológica Nacional and the Facultad Regional Delta [grant MAUTIDE0004482TC]. 